\title{
Eccentricity Dependence on Iron Abundance
}

\author{
Stuart F. Taylor \\ Participation Worldscope/Global Telescope Science \\ Sedona, Arizona, U.S., H. Kong \\ email: astrostuart@gmail.com
}

\begin{abstract}
The occurrence and eccentricity distribution of planets as a function of period is significantly different for iron-rich and iron-poor planet systems. We find that iron-poor stars with planets having periods between 525 and 600 days have higher eccentricity than such systems outside this range. If whole planet pollution causes the correlation of giant planet eccentricity with stellar iron abundance, then this cluster could be due to a paucity of pollution in this period range. Newly reported patterns of planet occurrence must result from planet system architectural features such as the snow line, followed by subsequent migration. Different results favor pollution or higher initial iron abundance causing the higher occurrence fraction of giant planets hosted by iron-rich stars, but the two explanations could be complementary. Relations between planet and stellar parameters are a major product of planet-finding, which promise further insights into star-planet system formation and evolution. Collaborators are sought to study these patterns. We expect a spirited debate over the relative contributions of initial abundances, disk accretion, and whole planet accretion.
\end{abstract}

Keywords. stars: abundances, planetary systems: dynamical evolution, tidal interactions, binaries (including multiple): close, planetary systems: formation, stars: fundamental parameters

\section{Introduction}

There has been a long debate over whether the correlation between iron abundance in stars $\left([\mathrm{Fe} / \mathrm{H}]_{*}\right)$ and giant planet occurrence is due to higher formation rates around ironrich stars, or if the stars were "polluted" by accretion of iron-rich disk material. Gonzalez (1997) cited the lack of a correlation of $[\mathrm{Fe} / \mathrm{H}]_{*}$ with the mass of the convective zone $(\mathrm{CZ})$ to reject the pollution hypothesis, but Murray \& Chaboyer (2002) showed that the then observed metallicities could be explained by the pollution of $\sim 5 M_{\oplus}$ into the convective zone (CZ) in combination with higher instrinsic metallicity and selection effects. Fischer \& Valenti (2005, hereafter FV05) ruled out that the occurrence-metallicity correlation is due to disk accretion by showing that there is no abundance variation of $\mathrm{Na}, \mathrm{Si}$, $\mathrm{Ti}$, or $\mathrm{Ni}$, etc. by condensation temperature as would be expected if dust grains were infalling.

Newly found correlations between $[\mathrm{Fe} / \mathrm{H}]_{*}$ and planet properties are presented as evidence that pollution from planets merging with stars makes a contribution to $[\mathrm{Fe} / \mathrm{H}]_{*}$. Such "whole planet" pollution would not be affected by stellar radiation, so would not vary by condensation temperature. Unexpected detail in planet numbers and eccentricity presented in T13b and here favor an explanation that whole planets pollute stars. The correlation of high iron abundance in binary SWPs (BSWPs) (Taylor 2013b; hereafter $\mathrm{T} 13 \mathrm{~b}$, and presentation at conference) suggests that in the presence of a binary companion, only in systems with higher initial iron can planet formation overcome the inhibiting effects of gravitational perturbations and irradiation.

It has been suggested (Dawson \& Murray-Clay 2013; hereafter DM13) that the correlation of iron-rich stars hosting planets with more highly eccentric orbits (T13b, Taylor 2012, 2013a; hereafter T12b, T13a) and with the presence or absence of the "hot Jupiter pileup" (DM13), could be due to planet-planet scattering being more frequent in the more crowded planetary systems formed from iron-rich systems (DM13). which could 

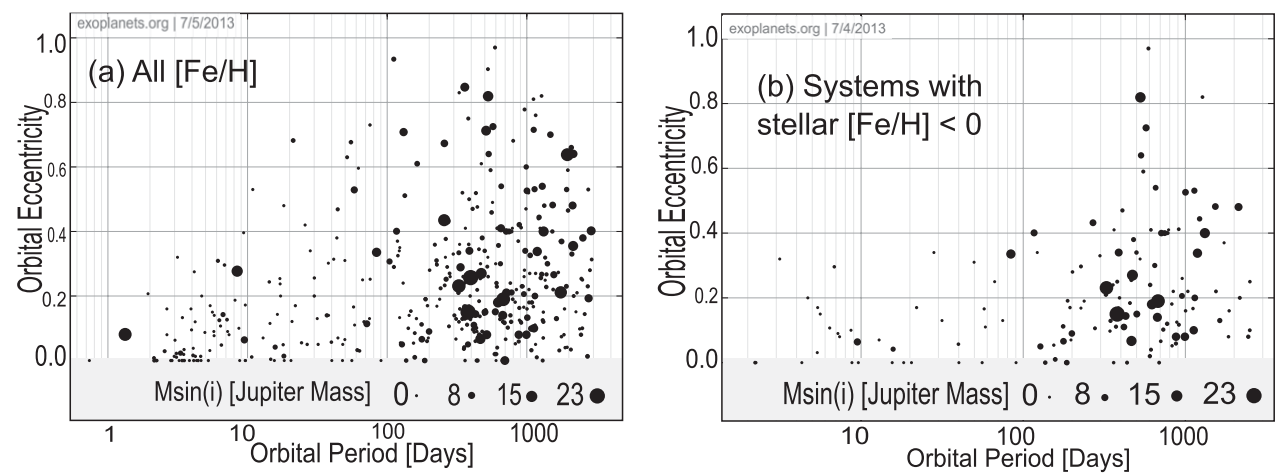

Figure 1. Eccentricity as function of period for systems found by radial velocity. This comparison of all systems (a) with low $[\mathrm{Fe} / \mathrm{H}]_{*}$ systems (b) shows that for most period ranges, high- $e$ systems are correlated with above-solar metallicity (a), but the low iron abundance systems (b) have a different pattern. We find a spike of five anomalously high-e systems confined within a narrow-period range, (b).

be due to higher iron formation rates, whole planet pollution, or both. The correlation of higher $[\mathrm{Fe} / \mathrm{H}]_{*}$ with binary SWPs (BSWPs) could be due to planet formation in the presence of a stellar companion requiring a higher initial iron abundance to overcome the inhibiting effects of gravitational perturbations and irradiation from the stellar companion. However, the eccentricity-period $(e-P)$ distribution of T13b varies with the period of the planet in a manner that likely reveals details of planet migration. To explain all of these, whole planet pollution could be further increasing the $[\mathrm{Fe} / \mathrm{H}]_{*}$ of systems crowded due to being preferentially initially metal-rich. If observed $[\mathrm{Fe} / \mathrm{H}]_{*}$ is due to both initial iron abundance and to pollution, it is important to determine the relative contributions.

\section{Unexpected dependence of $e-P$ on $[\mathrm{Fe} / \mathbf{H}]_{*}$ and binarity}

The eccentricity-period relation, shown in Fig. 1, can be seen to be very different for systems with $[\mathrm{Fe} / \mathrm{H}]_{*}$ below and above that of the sun. T13b and Taylor $(2013 \mathrm{c})$ also show that planets of single stars and binaries are separate populations. The eccentricity distribution as a function of period is described in T13b and Taylor (2013c), which show that iron-rich and poor populations below 500 days and above 600 days are different populations, with $99 \%$ and $96.5 \%$ probability respectively. Work using fewer statistics had not seen this, and said no correlation was evidence against pollution (e.g. FV05). There is a "spike" of iron-poor high eccentricity systems with periods from 520 to 600 days which are presented as evidence of pollution by T13b and Taylor (2013c).

\section{References}

Dawson, Rebekah I. \& Murray-Clay, Ruth A., 2013, ApJL, 767, L24.

Fischer, Debra A. \& Valenti, Jeff, 2005, ApJ, 622, 1102-1117.

Murray, N. \& Chaboyer, B., 2002, ApJ, 566, 442-451.

Gonzalez, Guillermo, 1997, MNRAS, 285, 403-412.

Taylor, S. F. 2012, arXiv:astro-ph/1211.1984.

Taylor, S. F. 2013a, arXiv:astro-ph/1301.4229.

Taylor, S. F. 2013b, arXiv:astro-ph/1305.5197.

Taylor, S. F. 2013c, extended version, to be submitted, arXiv:astro-ph. 